\title{
Refinamiento de la estrategia y búsqueda en diferentes bases de datos para una revisión sistemática
}

\author{
Improvement of the strategy and search in different databases for a \\ systematic review
}

\author{
Martha A. Sánchez-Rodríguez¹ y Osvaldo Daniel Castelán-Martínez² \\ ${ }_{1}^{1}$ Profesora de Tiempo Completo Titular C de la carrera de Química Farmacéutico Biológica, FES Zaragoza, UNAM. \\ ${ }^{1}$ Profesora de Tiempo Completo Asociado C de la carrera Química Farmacéutico Biológica, FES Zaragoza, UNAM
}

\section{INTRODUCCIÓN}

En un artículo publicado previamente en esta revista se mostró como hacer una búsqueda bibliográfica para una revisión sistemática (RS), enfocándose en cómo construir una estrategia básica a través de palabras clave, el uso de diversas bases de datos, la recuperación de la información y la construcción de la base de referencias para documentar la búsqueda. ${ }^{1}$ En este sentido, con el propósito de continuar con las estrategias de una búsqueda exhaustiva y precisa para para la investigación de una RS, se elaboró este texto en el que presentamos algunas recomendaciones para afinar la estrategia, para obtener la información relacionada con la pregunta de investigación y el uso de la nueva versión de Scopus, además de las indicaciones para continuar con la búsqueda en otras bases de datos (Web of Science, CENTRAL de Cochrane y Epistemonikos) y sitios web, con la finalidad de incrementar la información de potencial interés de acuerdo con el tema.

Recordemos que una RS es una investigación de tipo secundario debido a que toma la información de reportes primarios (experimentales u observacionales) orientados a una pregunta específica, resumiéndola y analizándola. Tiene dos modalidades, una de tipo cualitativo llamada exclusivamente revisión sistemática, y una de tipo cuantitativo denominada meta-análisis..$^{2,3}$ Un aspecto muy importante que se debe tomar en cuenta es que una buena revisión sistemática, con o sin meta-análisis, debe seguir los lineamientos de PRISMA (Preferred Reporting Items for Systematic Reviews and Meta-Analyses), cuya última versión es la 2020. En el punto 7 de la nueva lista de verificación (punto 8 de la versión 2009) se menciona "Presente las estrategias de búsqueda completas de todas las bases de datos, registros y sitios web, incluyendo cualquier filtro y los límites utilizados", 4,5 siendo uno de los aspectos que hace reproducible la investigación.

Al ser la información de los estudios el centro de análisis en este tipo de investigación documental, la búsqueda debe ser en diferentes bases de datos y lo más exhaustiva posible, ${ }^{1}$ pero tan bien dirigida que sea "manejable", es decir, obtener los reportes de investigación que permitan responder la pregunta de investigación, con una estrategia bien seleccionada, eliminando la información no relacionada con el objetivo del estudio, ni irrelevante. En 
este sentido, no existe un número mínimo ni máximo de documentos a incluir en una revisión sistemática, esto depende de la pregunta de investigación, lo que se ha publicado con respecto de esa pregunta, el objetivo y el alcance de la revisión; pero es obvio que si se recuperan miles de artículos científicos no se podrá leer, tal vez, ni el total de títulos, por ello es muy importante establecer una estrategia de búsqueda adecuada y repetirla, de ser posible, en todas las bases de datos consultadas para asegurar la reproducibilidad de la investigación. En ocasiones la estrategia final es muy grande, no obstante con la combinación de varios operadores boleanos, diferentes términos, comillas y filtros o límites utilizados en la búsqueda, se logrará mayor precisión, dependiente de la base de datos o sitio web consultados.

En este contexto, el objetivo de este documento es mostrar cómo se puede construir una estrategia de búsqueda más específica (refinada) para ser repetida en las diferentes bases de datos, partiendo de PubMed y la nueva versión de Scopus; además del uso de las bases Web of Science, CENTRAL de Cochrane y Epistemonikos, y algunos sitios web, para incrementar las opciones de búsqueda.

\section{Refinamiento de la estrategia de búsqueda}

Como se señaló en el artículo anterior, la estrategia de búsqueda comienza con las palabras clave seleccionadas de acuerdo con el problema de investigación, preferentemente en el orden de la estructura PICO, PEO, PECO, PICOST o como se haya formulado la pregunta de investigación, unidas con el conector AND. ${ }^{1}$ Las palabras clave pueden ser términos MeSH (Medical Subject Headings) en cuyo caso, después del término utilizado se incluirá entre corchetes el acrónimo, por ejemplo: phytoestrogens [MeSH]. EI MEDLINE de la Biblioteca Nacional de Medicina de EU (NLM) tiene un navegador MeSH (MeSH Browser) en donde se puede buscar si las palabras clave seleccionadas son términos $\mathrm{MeSH}$; si no aparece ningún resultado, el término no forma parte del vocabulario MeSH (Figura 1). ${ }^{6}$ La limitante del uso de términos MeSH en la estrategia de búsqueda es que excluye las referencias no indexadas por completo, las que están fuera del alcance y las que no incluyen esos términos en sus descriptores, ${ }^{7}$ además que no todos los buscadores de las diferentes bases de datos reconocen las palabras cuando se incluye el acrónimo. Son de utilidad cuando en la

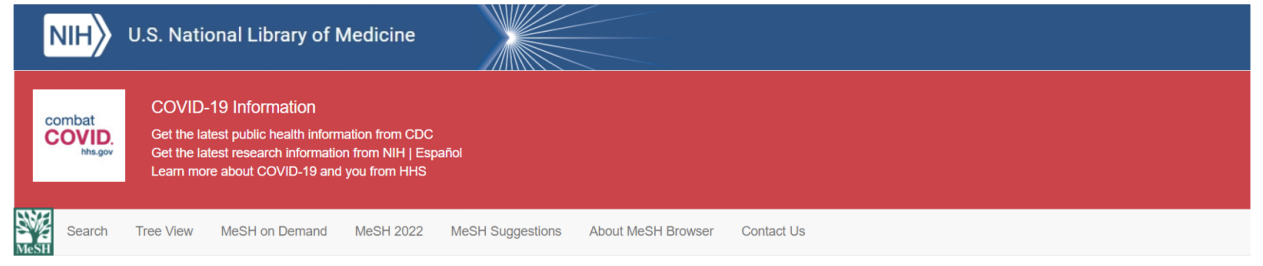

Medical Subject Headings 2021
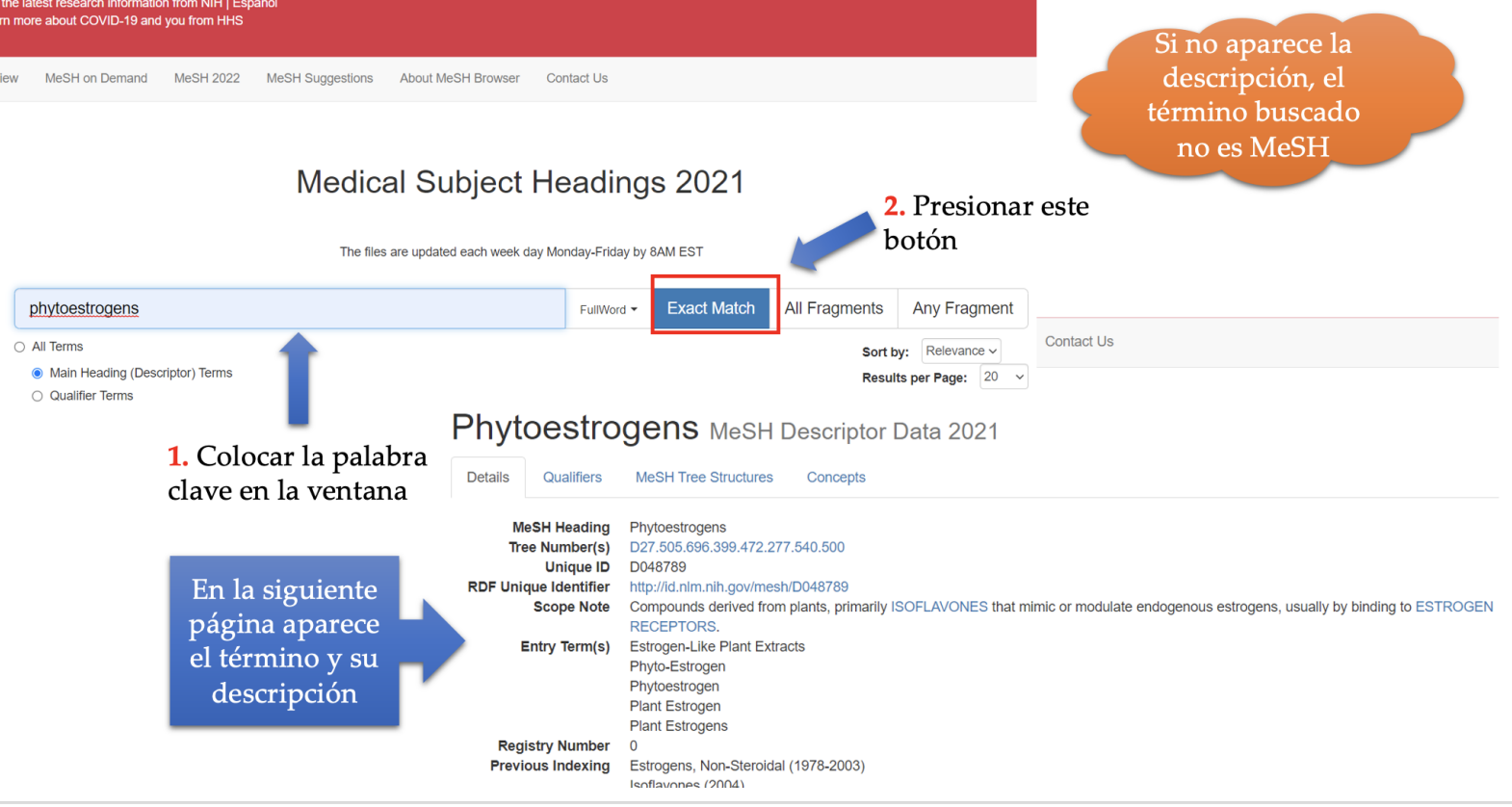

2. Presionar este botón

Phytoestrogens MeSH Descriptor Data 2021

Figura 1. Uso de la herramienta Medical Subject Headings de PubMed para la búsqueda de términos MeSH. Los números indican el orden de los pasos a seguir. 
búsqueda en PubMed se recupera mucha información y al hacer una revisión rápida nos damos cuenta de que no toda se ajusta a lo que requerimos.

Es posible combinar en una estrategia términos $\mathrm{MeSH}$ y no $\mathrm{MeSH}$, sobre todo si para la pregunta de investigación algunas palabras clave aún no son reconocidas en este tesauro o están relacionadas. Una vez seleccionados los términos a utilizar, se pueden añadir a la estructura base los sinónimos que se consideren importantes utilizando el operador de busqueda OR, encerrando entre paréntesis los términos que son equivalentes. Recordemos que el operador boleano OR es de suma, por lo que aumentará la información recuperada, ya que mostrará todos los artículos que tengan cualquiera de las palabras incluidas como sinónimos. ${ }^{8,9}$ Por ejemplo: (phytoestrogens OR isoflavones OR genistein), que también puede ser escrita con términos $\mathrm{MeSH}$ : (phytoestrogens [MeSH] OR isoflavones [MeSH] OR genistein $[\mathrm{MeSH}]$ ), recordando que con el uso de términos $\mathrm{MeSH}$ es menor la información recuperada.

En ocasiones, los términos que reflejan mejor una parte de la pregunta son dos o tres palabras que deseamos conformen uno solo. Si en la estrategia de búsqueda se incluyen esas palabras individualmente, aunque no estén ligadas con ningún operador boleano, las bases de datos pueden hacer la búsqueda como si fueran términos independientes, incrementando la cantidad de información recuperada, pero que puede no ser de utilidad. Para lograr que los buscadores tomen en cuenta todas las palabras como un solo término se pueden poner entrecomillas. Por ejemplo, si lo que se desea buscar es el efecto sobre la sintomatología menopáusica, al utilizar las palabras menopause symptoms podrán recuperarse artículos sobre menopausia y síntomas, pudiendo encontrar alguna información sobre síntomas que no tenga que ver con la menopausia; sin embargo, si se escriben las dos palabras entrecomillas: "menopause symptoms", los artículos reportados estarán relacionados con la sintomatología menopáusica.

Si después de utilizar estas recomendaciones la información recuperada contiene artículos que no son de interés (como revisiones, modelos animales, enfermedades no consideradas en la pregunta, etc.) se pueden utilizar los filtros o límites de cada base de datos, como fue señalado en el artículo previo, ${ }^{1}$ y/o utilizando el operador NOT. El operador NOT es de exclusión y puede agregarse a la estrategia de búsqueda cuando en una revisión rápida de la información recuperada nos damos cuenta de que hay alguna que no es de interés. ${ }^{1,8,9}$ Por ejemplo, si lo que nos interesa es hacer una revisión sobre el efecto de los fitoestrógenos comparado con los estrógenos sobre la sintomatología menopáusica, utilizando la estrategia como se ha ido señalando se encuentran algunos artículos en animales que no son de interés, por lo que, si se agrega al final de la estrategia NOT animals, las investigaciones con este tipo de modelos serán excluidas.

Una última recomendación cuando a pesar de todo lo anterior la información recuperada sigue siendo demasiada, es solicitar al buscador que las palabras clave sólo se encuentren en el título o el resumen. Esta acción se logra anexando a la estrategia el término [TIAB] al final; o que sólo esté en el título incluyendo [TI].

Reuniendo todas las recomendaciones señaladas anteriormente, la estrategia de búsqueda para la pregunta de investigación que estamos usando como ejemplo en PubMed quedaría como se muestra en la figura 2. Observe como va cambiando el número de artículos recuperados conforme se modifica la estrategia.

En la nueva versión de Scopus hay unas variantes en la escritura de la estructura. Inicialmente, toda la búsqueda la hace por título, resumen (abstract) y palabras clave (key) por lo que no es necesario el uso de TIAB ni que son términos MeSH. Reconoce los operadores boleanos, pero si no están dentro de un paréntesis deben escribirse junto con AND, por ejemplo, para indicar que no queremos reportes con animales la notación sería AND NOT animals, de otra forma hace la búsqueda diferente (Figura 3). En ocasiones marca error de sintaxis y no proporciona resultados, si sucede, salga de la plataforma y vuelva a entrar, aún está muy inestable.

\section{Base de datos Web of Science (WoS)}

Es una base de datos con información de investigación multidisciplinaria de alta calidad propiedad de Clarivate Analytics que requiere de suscripción, aunque se puede acceder a través de la Biblioteca Digital de la UNAM (BiDi), ingresando directamente o por medio de la Biblioteca de la FES Zaragoza, como se explicó anteriormente; ${ }^{1}$ recuerde que es necesario tener clave de la BiDi para poder utilizar estas bases de datos. Se incluyen revistas de ciencias biológicas, sociales, artes y humanidades por medio de las bases de datos: Science Citation Index expanded, Social Sciences Citation 


\begin{tabular}{|c|c|c|c|c|c|}
\hline Search & Actions & Details & Query & Results & Time \\
\hline$\# 9$ & $\cdots$ & $>$ & $\begin{array}{l}\text { Search: ("menopause symptoms" OR "climacteric symptoms") AND } \\
\text { (phytoestrogens [MeSH] OR isoflavones [MeSH] OR genistein } \\
\text { [MeSH]) AND (estrogens [MeSH] OR estradiol [MeSH]) NOT animals } \\
\text { [TIAB] Filters: Clinical Trial Sort by: Publication Date }\end{array}$ & 23 & $13: 02: 21$ \\
\hline \#8 & $\cdots$ & $>$ & $\begin{array}{l}\text { Search: ("menopause symptoms" OR "climacteric symptoms") AND } \\
\text { (phytoestrogens [MeSH] OR isoflavones [MeSH] OR genistein } \\
\text { [MeSH]) AND (estrogens [MeSH] OR estradiol [MeSH]) NOT animals } \\
\text { Filters: Clinical Trial Sort by: Publication Date }\end{array}$ & 22 & $13: 01: 33$ \\
\hline$\# 7$ & $\cdots$ & $>$ & $\begin{array}{l}\text { Search: ("menopause symptoms" OR "climacteric symptoms") AND } \\
\text { (phytoestrogens OR isoflavones OR genistein) AND (estrogens OR } \\
\text { estradiol) NOT animals Filters: Clinical Trial Sort by: Publication Date }\end{array}$ & 26 & 13:00:06 \\
\hline \#6 & $\cdots$ & $>$ & $\begin{array}{l}\text { Search: ("menopause symptoms" OR "climacteric symptoms") AND } \\
\text { (phytoestrogens OR isoflavones OR genistein) AND (estrogens OR } \\
\text { estradiol) NOT animals Sort by: Publication Date }\end{array}$ & 62 & $12: 59: 41$ \\
\hline \#5 & $\cdots$ & $>$ & $\begin{array}{l}\text { Search: ("menopause symptoms" OR "climacteric symptoms") AND } \\
\text { (phytoestrogens OR isoflavones OR genistein) AND (estrogens OR } \\
\text { estradiol) Sort by: Publication Date }\end{array}$ & 78 & $12: 57: 15$ \\
\hline
\end{tabular}

Figura 2. Estrategia de búsqueda utilizando operadores boleanos, términos MeSH y comillas para su uso en PubMed. La marcada con el \#5 es antes de poner filtros, la \#6 agregando el criterio de exclusión NOT animals; la \#7 seleccionando sólo ensayos clínicos; la \#8 con la misma estrategia, pero con el uso de términos MeSH; y en la \#9 se agregó el término TIAB. Observe como cambia el total de información recuperada.

\section{A. 119 document results

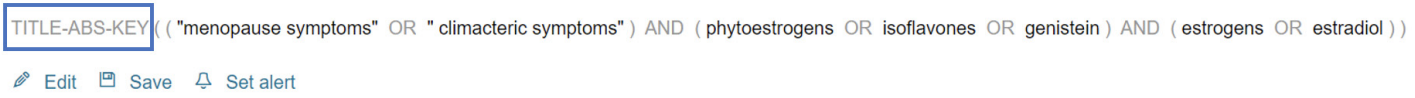

\section{B. 48 document results}

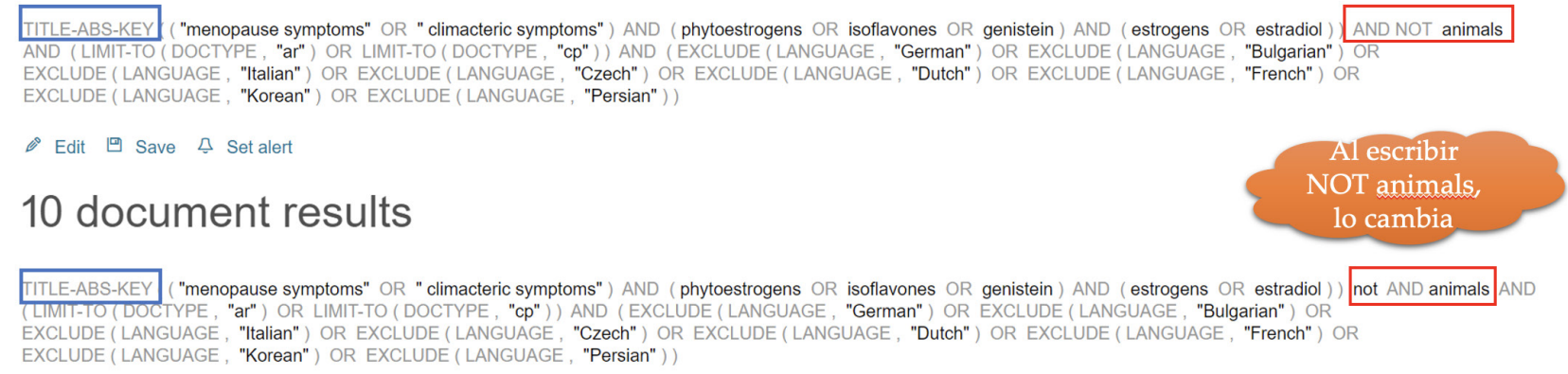

Figura 3. Reporte en Scopus aplicando la misma estrategia. El recuadro azul muestra como la búsqueda se hace por título, resumen y palabras clave (Title-Abs-Key). A. Sin filtros ni límites. B. Agregando límites por tipo de documento, idioma y no en modelos animales aplicando dos operadores juntos (AND NOT animals). C. Misma estrategia, pero escribiendo sólo NOT animals, la plataforma cambia la sintaxis. 
Index y Arts and Humanities Citation Index, con información en línea a partir de 1900 a la fecha y actualización semanal. ${ }^{10}$

Si el acceso se quiere hacer a través de la BiDi, se puede ingresar en la opción Ciencias Químico Biológicas y de la Salud. En esta sección se encuentran todas las bases de datos digitales a las que está suscrita la UNAM organizadas por orden alfabético, y hay varias colecciones de Web of Science, por lo que se debe tener cuidado en que sea la base de datos general y no alguna de sus colecciones particulares (Conference Proceedings Citation Index, Book Citation Index, Web of Knowledge: Science Citation Index, Zoological Records).

La nueva versión de esta base de datos ofrece la posibilidad de hacer las búsquedas con la versión anterior (Web of Science Classic) hasta que esté funcionando la plataforma nueva al 100\%. La versión 2021 da la opción de hacer la búsqueda en todas las bases de datos disponibles, además de las mencionadas se encuentran: Conference Proceedings Citation Index (Science y Social Sciences \& Humanities), Book Citation Index (Science y Social Sciences \& Humanities) y Emerging Sources Citation Index; dependiendo de la pregunta de investigación serán las bases a utilizar, pero los libros no son de utilidad y las revistas de artes y humanidades tal vez tampoco, por lo que podrían eliminarse antes de comenzar la búsqueda. Recuerde que los Proceedings o memorias de congreso son literatura gris, por lo que la revisión debe ser más cuidadosa, ${ }^{11}$ pero puede ser importante incluirla.

Hay algunas diferencias entre las dos versiones y como en este momento es opcional, revisaremos como se puede hacer una búsqueda en las dos. Los cambios no sólo son de apariencia, sino también en la forma de búsqueda y los resultados obtenidos.

La versión clásica la podemos consultar en español entrando por la BiDi; hace una búsqueda general por tema, localizando los términos en el título, resumen, palabras clave del autor y KeyWords Plus ${ }^{\circledR}$ (un base de datos propia de la plataforma). Tiene el operador AND implícito, de tal manera que no es necesario agregarlo, por lo que, siguiendo el ejemplo que hemos estado manejando podría escribirse: "menopause symptoms" phytoestrogens estrogens, y regresa el mismo resultado que si lo escribiéramos así "menopause symptoms" AND phytestrogens AND estrogens; los demás operadores boleanos funcionan como en PubMed; también reconoce las comillas para encontrar frases exactas (Figura 4). ${ }^{12}$ El formato de resultados es muy parecido Scopus, los filtros se encuentran del lado izquierdo y

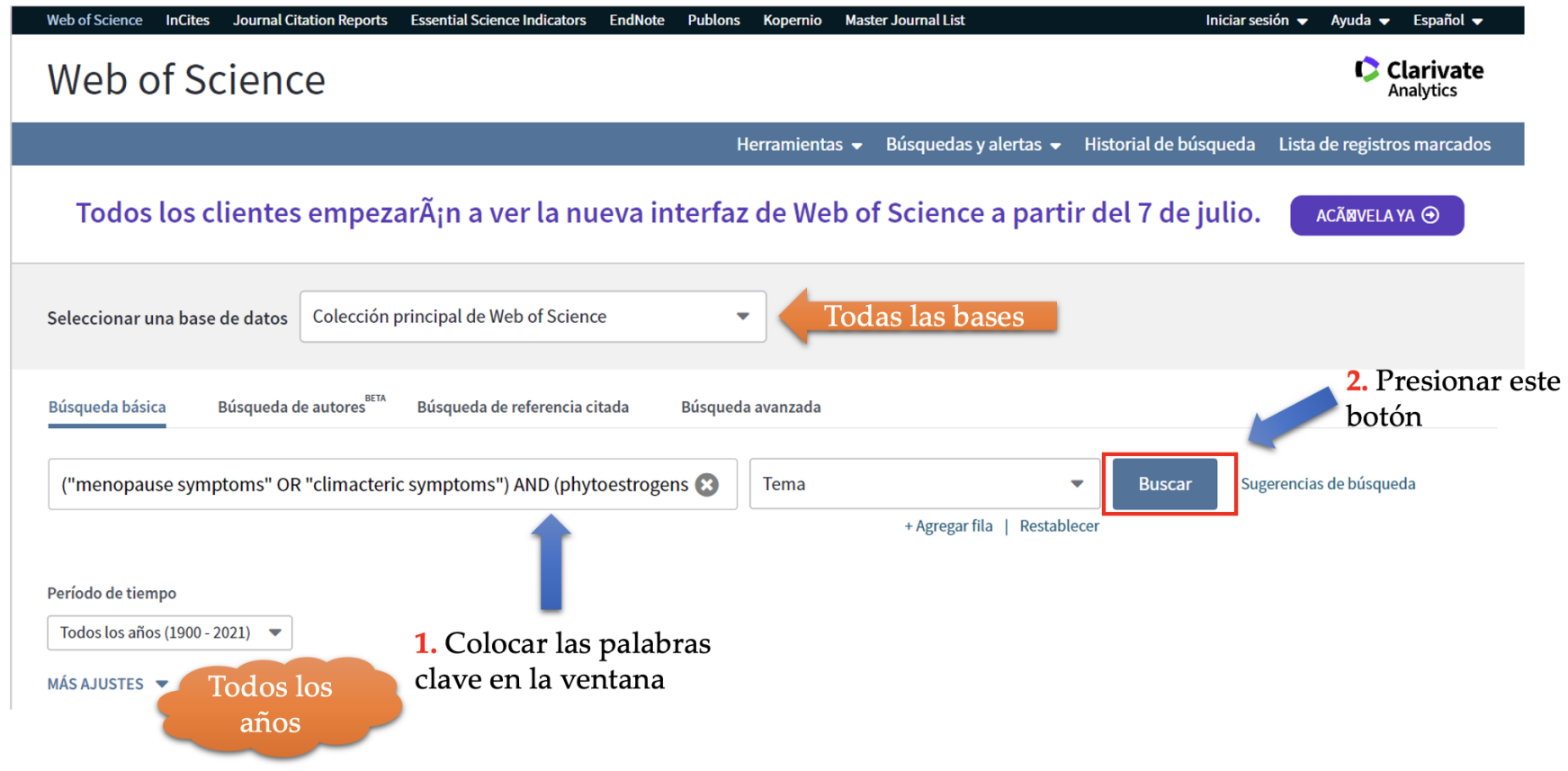

Figura 4. Búsqueda en la versión clásica de Web of Science. Los números indican el orden de los pasos a seguir. 


\section{Web of Science}

1) Clarivate

Analytics

\section{Buscar}

Herramientas - Búsquedas y alertas - Historial de búsqueda Lista de registros marcados

A.

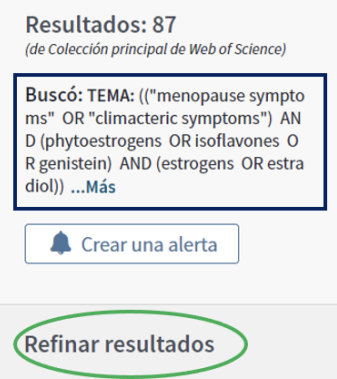

Ordenar por: Fecha $\underline{\underline{1}}$ Veces citado Conteo de uso Relevancia Más 1 de 9

(de Colección principal de Web of Science)

Buscó: TEMA: (("menopause sympto $\mathrm{D}$ (phytoestrogens OR isoflavones $\mathrm{O}$

$\square$ Seleccionar página $\square$ Exportar... Agregar a la lista de registros marcados

FI Analizar resultados

1. Effect of menopausal symptom treatment options on palpitations: a systematic review

Wl Crear informe de citas

Por: Sheng, Y.; Carpenter, J. S.; Elomba, C. D.; et ál.

Veces citado: 0

CLIMACTERIC

园 Acceso anticipado: AUG 2021

(en la Colección principal

de Web of Science)

Texto completo de la editorial Ver abstract $~-$

Conteo de uso

B. 78 resultados de Science Citation Index Expanded (SCI-EXPANDED), Conference Proceedings Citation Index - Science (CPCI-S):

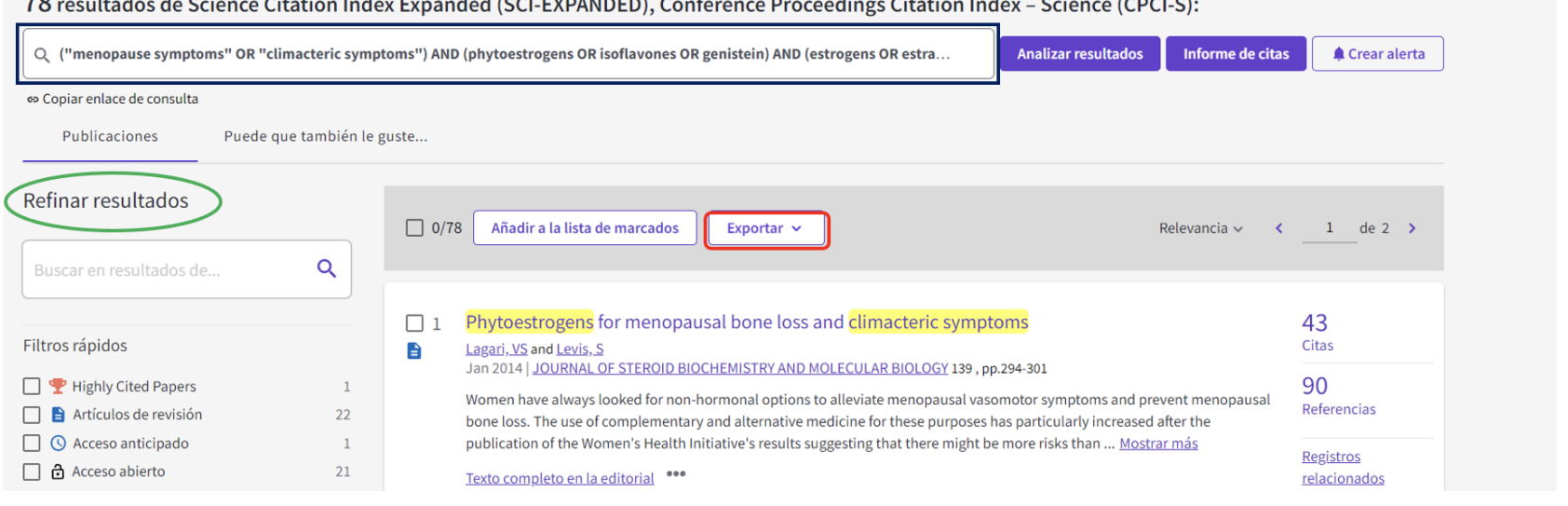

Figura 5. Reporte de resultados de una búsqueda en las dos versiones de Web of Science. A. Resultados en la versión clásica. B. Resultados en la nueva versión; nótese que disminuye el número de documentos recuperados utilizando la misma estrategia porque son bases de datos más específicas. El cuadro en azul marino señala la estrategia de búsqueda; el ovalo verde los filtros para refinar los resultados; y el cuadro rojo el botón para solicitar exportar los resultados en formato de Excel.

se puede seleccionar idioma, tipo de documento, fuente, área de publicación, etc.; además, ofrece la posibilidad de obtener texto completo de mucha de la información recuperada (Figura 5A).

La nueva versión se encuentra en diferentes idiomas y se puede seleccionar que la plataforma se muestre en español. Como se mencionó inicialmente, se han incluido diversas bases de datos y nos da la opción de seleccionar todas o sólo las que sean de interés de acuerdo con la pregunta de investigación (Figura 6). Si se limitan las bases de datos, se obtendrá una información más refinada, además de que se cuenta con los mismos filtros y la posibilidad de recuperación de texto completo como en la versión clásica. Una diferencia es que se localizan más fácilmente las bases de datos consultadas y la estrategia de búsqueda (Figura 5B). Ambas versiones brindan la posibilidad de exportar la búsqueda en una base de Excel directamente, sólo hay que seleccionar que se desea en ese formato y puede guardarse como se indicó previamente. ${ }^{1}$ La base de Excel tiene más campos que las otras bases de datos e incluye el resumen si el artículo lo tiene. 


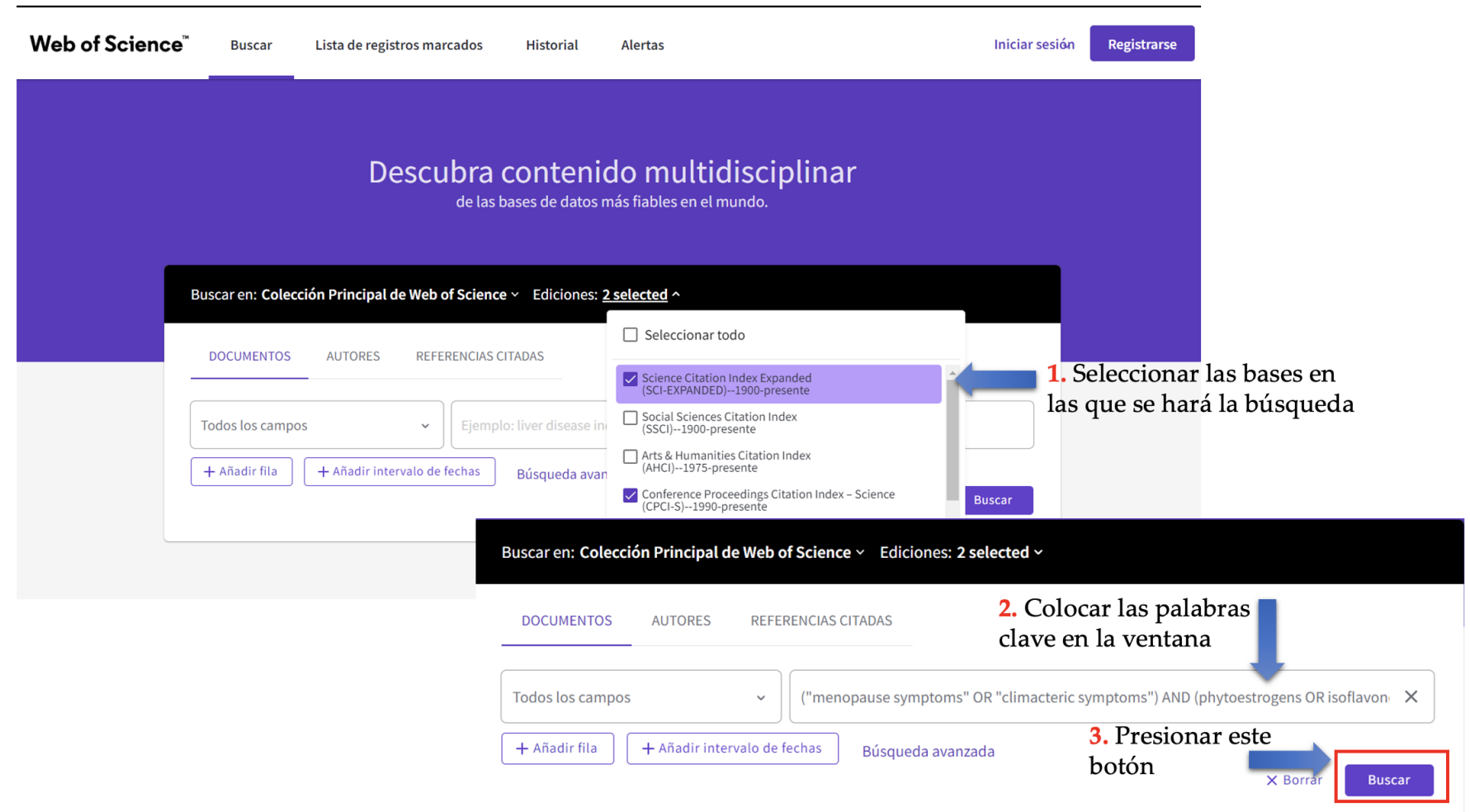

Figura 6. Búsqueda en la nueva versión de Web of Science. Los números indican el orden de los pasos a seguir.

\section{Cochrane Central Register of Controlled Trials (CENTRAL)}

Es la base de datos de ensayos clínicos aleatorizados y cuasialeatorizados de la Biblioteca Cochrane que es publicada por Wiley, por lo que es de suscripción. Hay la posibilidad de acceso a través de la Biblioteca Digital de la Facultad de Medicina de la UNAM utilizando clave $\mathrm{BiDi}$, encontrando el sitio en español, aunque no está habilitado todo el recurso.

La mayoría de los registros de CENTRAL provienen principalmente de PubMed, Embase y KoreaMed, además de otras fuentes como el sitio de registro de ensayos clínicos (Clinical Trials). Esta base contiene la información bibliográfica tradicional y en muchas ocasiones el resumen, pero no el texto completo. El espacio "Registros especializados" tiene una serie de informes de ensayos clínicos de importancia para algunos Grupos Cochrane y registros identificados por búsqueda manual en distintas fuentes biomédicas que no siempre está en otras bases de datos como PubMed, y tiene la ventaja de que, al hacer una búsqueda, todos los estudios son incluidos. ${ }^{13}$ Es importante señalar que cuando se consulta este recurso debe hacerse una buena revisión de la información obtenida porque pueden recuperarse protocolos de investigación que no deben ser incluidos en la revisión sistemática.

Como se comentó en un inicio, para acceder a esta base de datos es recomendable hacerlo a través de la Biblioteca Digital de la Facultad de Medicina de la UNAM, ya que tiene el acceso directo a la Biblioteca Cochrane. En el sitio Cochrane, se debe abrir el menú Ensayos y ahí seleccionar Buscar ensayos (CENTRAL) como se muestra en las figuras $7 \mathrm{~A}$ y $\mathrm{B}$.

Al encontrarnos en el sitio de CENTRAL, verificar que en el buscador esté indicado Título Resumen Palabras clave, para que en esas condiciones se realice la exploración, si no lo está, abrir el menú y seleccionarlo (Figura $8 \mathrm{~A})$. Posteriormente, escribir en la ventana de búsqueda la estrategia. La plataforma mostrará los resultados de la búsqueda, separándola en diferentes pestañas de acuerdo con la clasificación de la información; en el caso que nos ocupa, solamente revisaremos la pestaña de Ensayos (Figura 8B). Los filtros de este sitio sólo son: año de primera publicación, fecha de registro en 


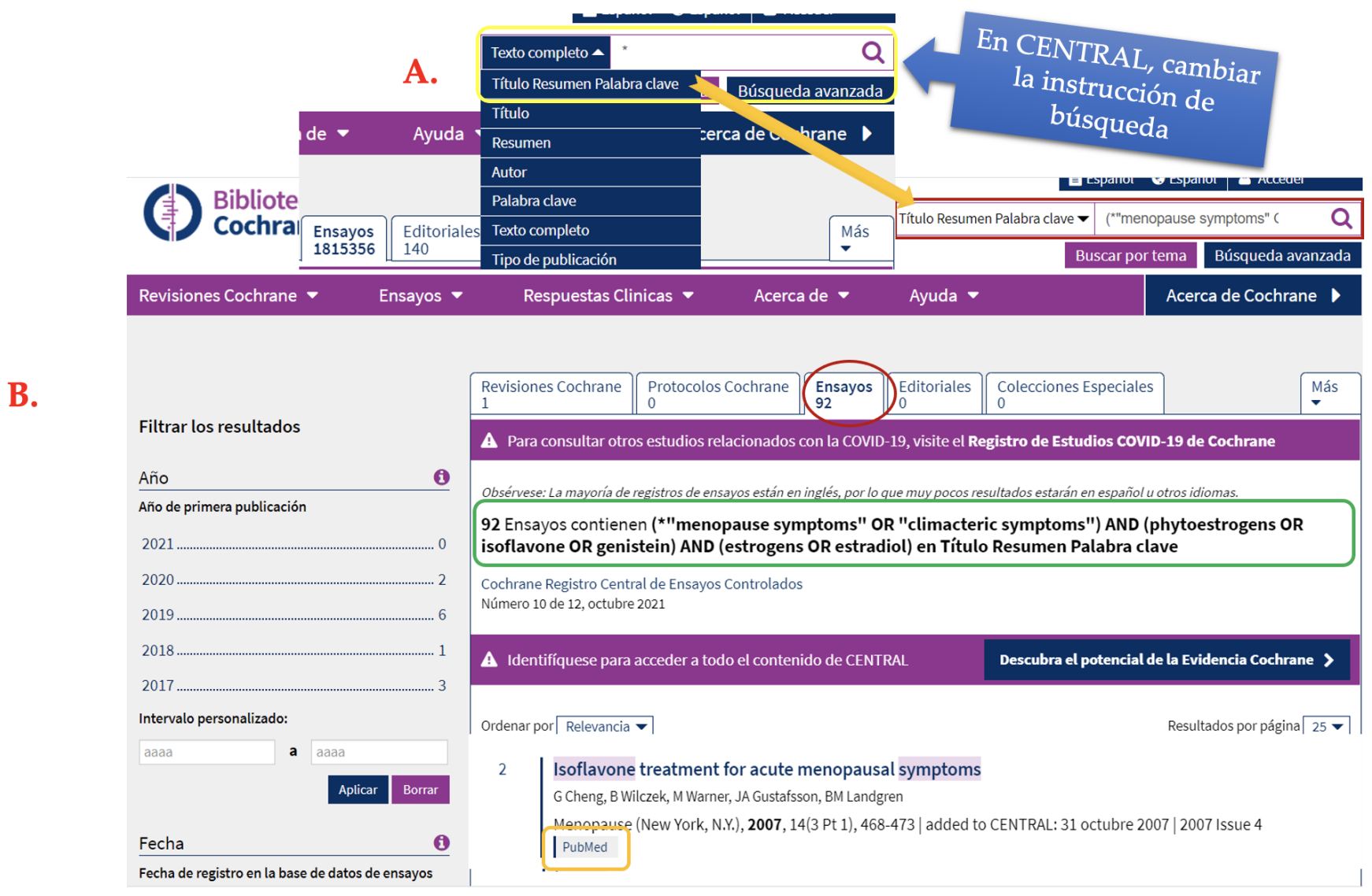

Figura 8. Búsqueda y reporte de resultados en CENTRAL de Cochrane. A. Seleccionar la forma de búsqueda y escribir en la ventana la estrategia. B. Resultados, ir a la pestaña de Ensayos (círculo rojo). Se nos indica el total de documentos que cumple con la estrategia propuesta (cuadro verde) y base de datos en donde se localiza el documento (cuadro naranja).

la base de datos de ensayos CENTRAL y fuente, por lo que no es necesario utilizarlos. Lamentablemente, el uso del sitio está limitado, por lo que, sí podemos hacer la búsqueda, pero no nos permite obtener el listado de referencias y en ocasiones tampoco el resumen, de ahí que la lectura de títulos tiene que hacerse directo de la pantalla y tomar los datos de las referencias que consideramos pueden ser de utilidad para la revisión que se está realizando. Una ventaja es que nos indica la base de datos en la que se encuentra, por lo que es posible eliminar desde ahí lo que está en PubMed (sin olvidar tomar el dato para el registro total) y tratar de localizar los documentos restantes de interés por otros medios, como se comentó anteriormente. ${ }^{1}$

\section{EPISTEMONIKOS}

Es una base de datos colaborativa multilingüe de evidencia en salud de acceso libre. Se anuncia como la mayor fuente de revisiones sistemáticas relevantes y artículos originales para la toma de decisiones en salud, por lo que es una fuente de otros tipos de evidencia científica. Su objetivo es recopilar información científica relevante para la toma de decisiones en salud, y proveer un acceso rápido y confiable a la mejor evidencia disponible para preguntas de contextos reales. El nombre de esta base de datos es una palabra griega que significa "lo que vale la pena conocer ", que en latín se tradujo como scientia. ${ }^{14}$

Funciona en nueve idiomas: alemán, árabe, chino, francés, holandés, inglés, italiano, español y portugués, y todos los artículos son traducidos a los nueve idiomas por un software integrado en la web.

El acceso puede ser directo con esta liga https://www. epistemonikos.org/es\#; o a través del blog de la Biblioteca Campus I de la FES Zaragoza como se mostró en el artículo anterior. ${ }^{1}$ Para la búsqueda se coloca en la ventana los términos seleccionados, siguiendo la sintaxis que hemos visto para las otras bases de datos. 


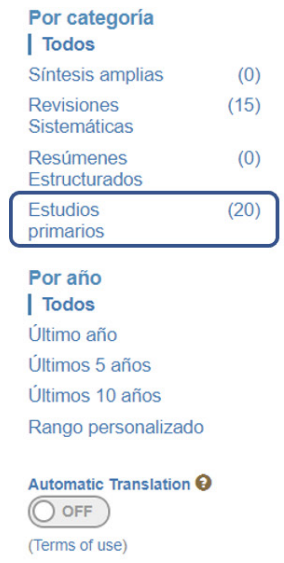

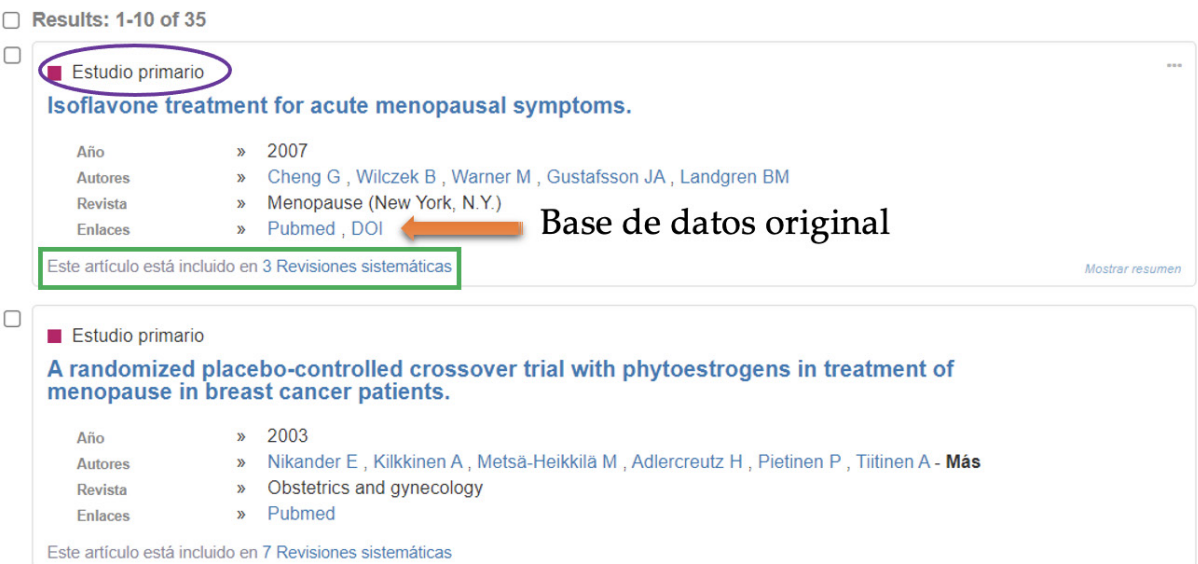

Figura 9. Formato de resultados de Epistemonikos. En la ventana superior (cuadro rojo) se muestra la estrategia de búsqueda. Del lado izquierdo se encuentra el tołal de información recuperada separada por tipo de artículo, lo que interesa son los estudios primarios (cuadro azul marino). En los resultados propiamente, está el tipo de artículo (círculo morado), la base de datos en donde se encuentra originalmente (flecha naranja), y si ha sido utilizada la información en alguna revisión sistemática (cuadro verde).

Permite el ingreso de palabras en diferentes idiomas, incluso mezcladas, pero para un mejor resultado se recomienda que todos los términos estén en inglés. Los resultados se presentan por relevancia de la representatividad de los términos buscados en la información recuperada, ${ }^{15}$ semejante a PubMed. La información se encuentra separada en revisiones sistemáticas y estudios primarios (artículos originales), lo que facilita la selección de la información; además, muestra la base de datos en la que se encuentra el artículo originalmente y si la información ha sido utilizada en alguna revisión sistemática (Figura 9). La limitante es que se tienen que tomar los datos de forma manual pues no hay forma de obtener un archivo digital como en las otras bases de datos.

\section{Otras bases de datos}

Hemos revisado en el artículo previamente publicado y en el actual, las bases de datos más importantes que deben consultarse, y tenemos acceso en la UNAM, para realizar una revisión sistemática en las ciencias químicobiológicas y de la salud; sin embargo, pensando en una revisión exhaustiva y que es posible que exista información que no se encuentra en esas bases de datos, se puede continuar la búsqueda en otros sitios web que también tienen recopilación de documentos científicos, pero que no están diseñados para este tipo de búsquedas, por lo que son menos selectivos. La revisión de los resultados de la búsqueda debe realizarse en línea, así como tomar los datos de las referencias útiles manualmente, ya que no hay posibilidad de obtener un archivo de resultados.

Estos sitios web son menos estrictos en la selección de la información que los conforman, por lo que se corre el riesgo de encontrar algunos documentos con baja calidad científica y editorial, con errores metodológicos, pero no se puede generalizar. Esto significa que hay que ser más cuidadosos en la lectura de los que tengan la posibilidad de ser incluidos en la revisión sistemática. De los diversos sitios que existen en la web, los que contienen una vasta información con la ventaja de que es Latino e Iberoamericana, son Dialnet, Redalyc, Medigraphic e IMBIOMED, todos de acceso libre, aunque la última requiere de un registro y pregunta para qué se utilizará la información.

Dialnet es un repositorio de la Universidad de la Rioja que contiene revistas, tesis y memorias de congresos 


\section{A. O Dialnet}

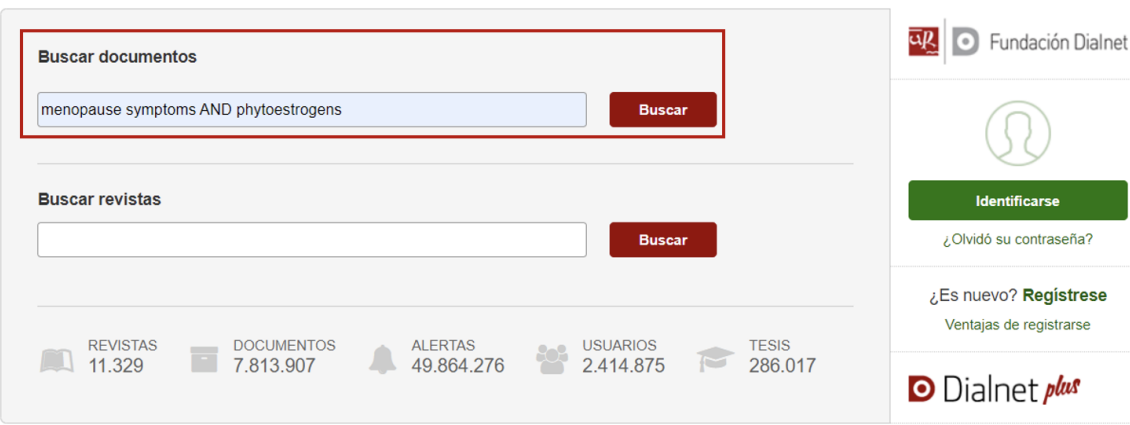

B.

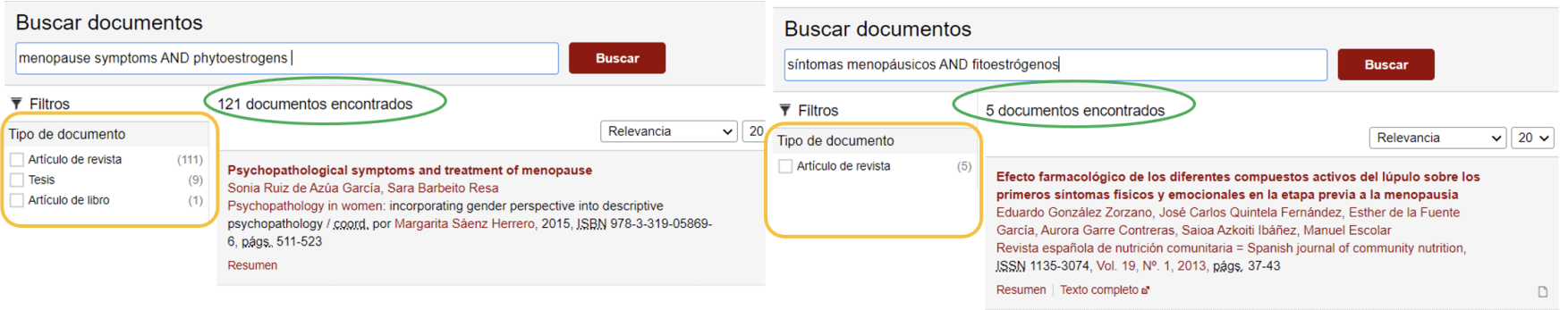

Figura 10. Búsqueda y reporte de resultados de Dialnet. $A$. La búsqueda debe realizarse en la ventana de Buscar documentos con no más de dos términos unidos por AND (cuadro rojo). B. El reporte de resultados es diferente si se utilizan palabras en inglés (lado izquierdo) o español (lado derecho). En ambos se observa el total de documentos recuperados encerrados con el óvalo verde, el tipo de documento y el total de cada uno (cuadro naranja).

de diversas áreas del conocimiento: ciencias básicas y experimentales, geociencias, ciencias biológicas, agricultura y alimentación, tecnología, economía, ciencias jurídicas, ciencias sociales, psicología y educación, humanidades, arte y filologías. La mayoría de las revistas incluidas tienen disponibilidad de texto completo principalmente en inglés y español. Es posible ingresar al sitio por medio del blog de búsqueda de información campus I de la Biblioteca de la FES Zaragoza, encontrándose en la sección de Regionales de Enfermería y Psicología.

La búsqueda debe hacerse en la ventana de Buscar documentos (Figura 10A), y al ser un recurso limitado, si se utiliza la estrategia completa como en las otras bases de datos, es muy posible que el resultado sea cero, ya que no tiene la posibilidad de manejar muchos términos. Se sugiere utilizar sólo dos términos unidos por el operador AND, pudiendo estar las palabras en inglés o español. Si éstas se usan en los dos idiomas, se pueden recuperar documentos diferentes, los resultados se muestran organizados por orden de relevancia (Figura 10B).
Redalyc es la red de revistas científicas de acceso abierto no comercial propiedad de la academia manejada por la Universidad del Estado de México, que incluye materiales de ciencias sociales, ciencias naturales y exactas, multidisciplinarias, arte y humanidades de Latinoamérica.

Para la búsqueda, los términos deben estar en español, no reconoce los operadores boleanos, pero se puede utilizar la combinación de dos palabras con una "y". Es posible utilizar los términos compuestos entrecomillas, por ejemplo, "síntomas menopáusicos" (Figura 11A), esto último favorece el refinamiento de la búsqueda, de otra manera, los resultados arrojan mucha información que no será de utilidad. Tiene la posibilidad de filtrar la información por año, disciplina y país; de lo cual, se recomienda usar sólo el filtro de disciplina si es que hay algunas referencias que no son de interés para la pregunta de investigación (Figura 11B). Toda la información recuperada está disponible para ser consultada en texto completo. 
A.

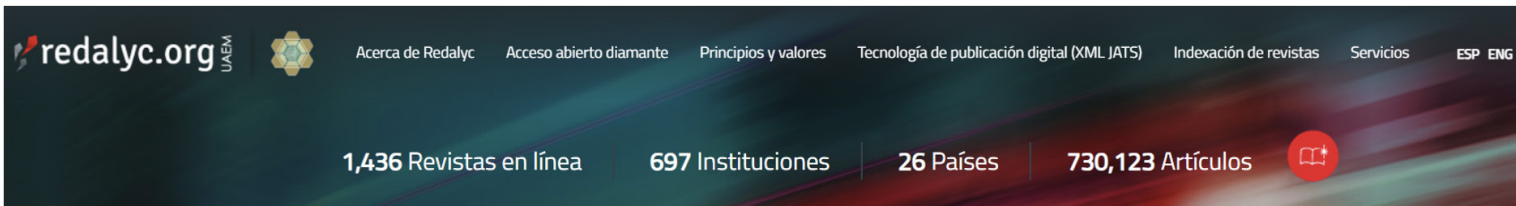

\section{Buscar en redalyc}

\begin{tabular}{|lll|}
\hline Artículos & $\checkmark$ & "sintomas menopáusicos" y fitoestrógenos \\
\hline
\end{tabular}

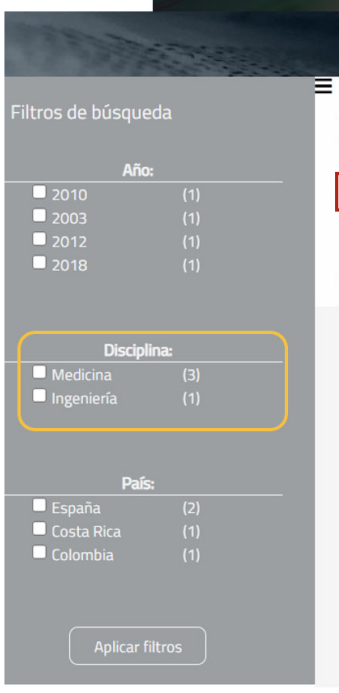

B. Búsqueda de artículos

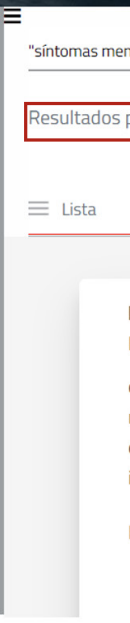

Revista Clínica de Medicina de Familia, 2010,3(3)

carácter de terapia "natural". ASPECTOS HISTÓRICOS DE LOS FITOESTRÓ- GENOS La historia natural de los fitoestrógenos como remedios terapéuticos es... bien, el interés científico por el análisis y estudio de los fitoestró- genos y sus efectos tendrá su origen en 1982 con Axelson y en 1984 con... de mujeres menopáusicas de nuestro país en comparación con mujeres de países asiáticos, en donde la ingesta de fitoestró- genos, concretamente de

Resumen: $\square$

$囚 \mathrm{PDF}$

Figura 11. Búsqueda y reporte de resultados en el sitio Redalyc. A. Se deben escribir los términos en español, incluyendo los conectores. B. En los resultados aparecen los términos utilizados (cuadro rojo), el total de información recuperada (cuadro verde) y de las referencias se tiene la posibilidad de obtener el texto completo (flecha naranja). En los filtros, se recomienda utilizar sólo los de disciplina (cuadro naranja en el lado izquierdo), si fuera necesario.

Los otros sitios web; Medigraphic (https://www.medigraphic.com/newMedi/) e IMBIOMED (https://www.imbiomed.com.mx/) son más bien repositorios comerciales de revistas de literatura biomédica cuyos artículos no tienen costo, ambos son de México y tienen muchas revistas en común; además, están organizados por nombre de revista y campo de especialidad médica, y son una opción cuando se quiere tener información de México y Latinoamérica.
Al ser repositorios, la estrategia de búsqueda como la hemos utilizado no funciona. Reconocen las palabras en español, no identifican los conectores boleanos ni en inglés ni en español, en muchas ocasiones no es posible poner palabras combinadas porque el resultado de la búsqueda será muy pequeño o cero. Medigraphic agrega una simbología exclusiva para mejorar una búsqueda (cuadro 1). ${ }^{16}$

\section{Cuadro 1. Simbología particular utilizada en el sitio web Medigraphic para afinar la búsqueda.}

Símbolo Que significa $\quad$ Ejemplo

+ La búsqueda debe contener la palabra después del símbolo

- $\quad$ La búsqueda debe excluir la palabra después del símbolo

* $\quad$ Trunca la palabra y se reconoce como raíz

Comillas Reconoce una frase

\author{
+menopausia \\ -cáncer \\ osteop* \\ losteoporosis, \\ osteopenia, etc.) \\ "síntomas \\ menopáusicos"
}


Tienen el inconveniente de que no toda la información recuperada es de utilidad para la pregunta de investigación, y la revisión de la información debe hacerse "manualmente" porque no hay manera de obtener un archivo digital, de ahí que, si algún registro es de utilidad, deberán capturarse los datos principales directamente en la base de referencias que se esté formando.

Finalmente, hemos hecho un recorrido por diferentes bases de datos y sitios web para la obtención de documentos para llevar a cabo una revisión sistemática basada en una pregunta de investigación; sin embargo, la información presentada en estos artículos puede ser de utilidad para cualquier tipo de búsqueda bibliográfica, por ejemplo, la construcción de un marco teórico o la discusión de un reporte de investigación (tesis o artículo científico), con la ventaja de que lo recuperado será específico en función de la estrategia de búsqueda utilizada. La diferencia entre estas dos opciones es que la última no requiere del registro de la información obtenida, la estrategia de búsqueda ni la recuperación de las bases de datos.

\section{AgradeCIMIENTOS}

El manuscrito fue revisado y editado en el Programa para la Investigación Bibliográfica Científica sobre Salud (PIBCIS) de la FES Zaragoza, UNAM. El artículo es parte del material de apoyo didáctico elaborado por la Red Académica Asesora de Revisiones Sistemáticas de la FES Zaragoza, UNAM (RAARS), financiada por la Dirección General de Asuntos del Personal Académico, UNAM, proyecto PAPIME PE203421.

\section{Referencias}

1. Sánchez-Rodríguez MA. Estrategias para la búsqueda de información bibliográfica científica para una revisión sistemática. CyRS. 2020; 2(2): 71-88.

2. Beltrán OA. Revisiones sistemáticas de la literatura. Rev Colomb Gastroenterol. 2005: 20(1): 60-69.

3. Aguilera-Eguía R. ¿Revisión sistemática, revisión narrativa o metaanálisis? [Carta] Rev Soc Esp Dolor. 2014; 21(6): 359-360.

4. Moher D, Liberati A, Tetzlaff J, Altman DG, The PRISMA Group. Preferred Reporting Items for Systematic Reviews and Meta-Analyses: The PRISMA statement. PLoS Med. 2009; 6(7): e1000097. doi: 10.1371/journal.pmed1000097.

5. Page MJ, McKenzie JE, Bossuyt PM, Boutron I, Hoffmann TC, Mulrow CD, et al. The PRISMA 2020 statement: an updated guideline for reporting systematic reviews. BMJ 2021; 372: n71. doi: 10.1136/bmj.n71

6. National Library of Medicine. Finding terms to describe subject content. [Consultado: 15 octubre 2021] Available from:

https://www.nlm.nih.gov/bsd/disted/meshtutorial/principlesofmedlinesubjectindexing/findingterms/index.html

7. National Library of Medicine. Searching PubMed using MeSH search tags. [Consultado: 15 octubre 2021] Available from:

https://www.nlm.nih.gov/bsd/disted/meshtutorial/searchingpubmedusingmeshtags/index.html

8. Aleixandre-Benavent R, González-Alcaide G, González de Dios J, Alonso-Arroyo A. Fuente de información bibliográfica (I). Fundamentos para la realización de búsquedas bibliográficas. Acta Pediatr Esp. 2011; 69(3): 131-136.

9. Rivas-Ruiz R, Talavera JO. Investigación clínica VII. Búsqueda sistemática: como localizar artículos. Rev Med Inst Mex Seguro Soc. 2012; 50(1): 53-58.

10. Clarivate Analytics. Web of Science. [Consultado: 16 octubre 2021] Available from: https://www.bidi.unam.mx/ index.php/cobertura-tematica/ver-todos-los-recursos/724web-of-science-full.

11. Lauper M. ¿Qué hacer con la literatura gris? [Editorial] Interciencia. 2007; 32(1): 5.

12. Web of Science. Buscar en el campo tema. [Consultado: 16 octubre 2021] Available from: http://images.webofknowledge.com.pbidi.unam.mx:8080//WOKRS535R111/ help/es_LA/WOS/hs_topic.html.

13. Biblioteca Cochrane. Registro Cochrane Central de Ensayos Controlados (CENTRAL). [Consultado: 17 octubre 2021] Available from: https://www.cochranelibrary.com/es/ central/about-central

14. Epistemonikos. Sobre la base de datos Epistemonikos. [Consultado: 19 octubre 2021] Available from: https://www. epistemonikos.org/es/about_us/who_we_are

15. Epistemonikos. Como se usa. [Consultado: 19 octubre 2021] Available from: https://www.epistemonikos.org/es/ about_us/how_to_use

16. Medigraphic. Mejora tus resultados usando estos símbolos. [Consultado: 21 octubre 2021] Disponible en: https:// www.medigraphic.com/newMedi/buscar/buscador.php 